\title{
PULSATION OF ROTATING AND MAGNETIC STARS
}

\author{
SERGEI V. VORONTSOV \\ Institute of Astronomy and Department of Applied Mathematics and \\ Theoretical Physics, University of Cambridge, Cambridge CB3 0HA, \\ England, and \\ Institute of Physics of the Earth, Moscow 123810, Russia \\ (permanent address)
}

\begin{abstract}
Rotation and a magnetic field break the spherical symmetry of a star viewed as a pulsating system, lifting the degeneracy of oscillation frequencies, and leading to (sometimes prominent) observational consequences. Theoretical studies of rotational and magnetic effects in pulsating stars are reviewed, starting with simple configurations with slow rotation and weak magnetic fields.
\end{abstract}

\section{INTRODUCTION}

The theoretical oscillation spectrum of a spherically symmetric, non-rotating and non-magnetic star is largely degenerate. The nature of this degeneracy is made transparent by the simple considerations about the completeness property of the oscillation spectrum of the spherically symmetric star.

An arbitrary displacement field in a spherical domain occupied by the star can be decomposed in terms of spheroidal vector spherical harmonics

$$
\mathbf{u}_{\ell m n}(\mathbf{r})=U_{\ell m n}(r) \hat{r} Y_{\ell m}(\theta, \phi)+V_{\ell m n}(r) \nabla_{1} Y_{\ell m}(\theta, \phi),
$$

and toroidal vector spherical harmonics

$$
\mathbf{u}_{\ell m n}(\mathbf{r})=-W_{\ell m n}(r) \hat{r} \times \nabla_{1} Y_{\ell m}(\theta, \phi),
$$

where $\nabla_{1}$ is angular part of gradient operator

$$
\nabla_{1}=\hat{\theta} \frac{\partial}{\partial \theta}+\frac{1}{\sin \theta} \hat{\phi} \frac{\partial}{\partial \phi}
$$

and $\hat{r}, \hat{\theta}, \hat{\phi}$ are unit vectors in a spherical coordinate system. In linear analysis, the time dependence is separated by a common factor $\exp (i \omega t)$.

The usual $\mathrm{p}, \mathrm{f}$ and $\mathrm{g}$ modes represent the spheroidal oscillations. Because of the spherical symmetry, their frequencies are degenerate with respect to the azimuthal order $m$.

Toroidal fields are horizontal and divergence-free (solenoidal). They describe torsional oscillations, with restoring forces provided by the shear stresses. With the only exception of neutron stars (McDermott, Van Horn and 
Hansen 1988; Strohmayer et al. 1991), the shear stresses actually produce no restoring forces, and frequencies of the torsional oscillations are zero ("trivial modes"). Formally, these modes describe an arbitrary time-independent differential rotation.

In the spectrum of spheroidal oscillations, there is also one "trivial mode" with zero frequency, which is the fundamental mode of degree $\ell=1$. This mode describes the free motion of the star as a whole. The complete oscillation spectrum thus describes all the possible motions of the star in free space: the motion of the star as a whole, its arbitrary free rotation, and oscillations around the equilibrium.

Rotation and a magnetic field make the oscillation spectrum much reacher. They destroy the spherical symmetry, lifting the degeneracy of the frequencies of $p, f$ and $g$ modes. The splitting of the frequency multiplets with respect to the azimuthal order $m$ can be measured and used for the diagnostics of the internal rotation and the internal magnetic field. In addition to pressure perturbations, which provide the restoring forces for $p$ modes, and buoyancy forces, which are the restoring forces for $g$ and $f$ modes, the rotation and magnetic field provide new restoring forces, which are Coriolis forces and Lorentz forces, leading to the appearance of non-trivial modes of quite different physical nature.

Pulsations of rotating and magnetic stars were an object of intensive study for decades, starting with pioneering works of Cowling and Newing (1949) and Ledoux (1951). There are a number of related topics, including stability of differentially rotating stars, angular momentum transport and interaction between rotation, pulsation and convection, which cannot be covered by this short review. For a more extensive bibliography of current literature, and for the early work, the reader is referred to the monographs of Cox (1980) and Unno etal. (1989), and to the papers cited below.

\section{SLOW ROTATION}

The influence of rotation on $\mathrm{p}, \mathrm{f}$ and $\mathrm{g}$ modes can be studied using perturbation analysis, if this influence is small. "Slow" rotation means that the Coriolis forces are small compared with inertial forces, i.e. the angular velocity of rotation is small compared with oscillation frequencies, and the rotational distortion of the equilibrium configuration is small, i.e. centrifugal forces are small compared with gravity.

The rotational effects on the oscillation frequencies are usually analysed for the case of axisymmetric rotation, $\Omega=\Omega(r, \theta)$. An interesting situation when the stellar core and the envelope rotate around different axes, and the possibility of detecting such a rotation in the oscillation data, is discussed in these proceedings by Gough and Kosovichev (1992).

\section{First-order effects of rotation}

The leading-order effects on the oscillation frequencies are linear in $\Omega$, and independent of the rotational distortion of the equilibrium stellar configuration, which is of order $\Omega^{2}$. The perturbation analysis is thus straightforward, and does not require any additional assumptions. 
A general expression for the frequency perturbations due to an arbitrary axisymmetric differential rotation $\Omega=\Omega(r, \theta)$ was first obtained by Hansen, Cox and Van Horn (1987):

$$
\delta \omega=-\frac{(\mathbf{u}, \Omega[m-i \hat{z} \times] \mathbf{u})}{(\mathbf{u}, \mathbf{u})},
$$

where $\hat{z}$ is unit vector in the direction of rotation, which is taken also to be the direction of $z$-axis of the spherical coordinate system, $\mathbf{u}$ is the zero-order displacement field, and scalar product is defined by

$$
\left(\mathbf{u}_{1}, \mathbf{u}_{2}\right)=\int \rho_{0} \mathbf{u}_{1}^{*} \cdot \mathbf{u}_{2} d v
$$

the integral is taken over the spherical volume occupied by the star, $\rho_{0}$ is the equilibrium density and an asterisk denotes complex conjugate. By separating angular dependences, the volume integrals in the expression (4) can be reduced to one-dimensional integrals containing $U(r)$ and $V(r)$ (for the $\theta$-dependence of $\Omega(r, \theta)$ expressed in terms of harmonic functions, see Cuypers 1980$)$.

Below in this section, we discuss the response of the oscillation frequencies to differential rotation, and thus the diagnostic capability of frequency measurements in studying internal stellar rotation. For convenience the angular velocity distribution expanded in terms of spherical harmonics

$$
\Omega=\sum_{i} \Omega_{i}(r) Y_{i 0}(\theta, \phi)
$$

Such a representation of $\Omega(r, \theta)$ is a natural choice when working in spherical geometry, and appears to be the most convenient for the practical inversions of rotational splitting (Durney, Hill and Goode 1988; Gough and Thompson 1990). The frequency perturbation becomes (Vorontsov 1981)

$$
\begin{aligned}
\delta \omega & =\sum_{i} \int_{0}^{R} \rho_{0} r^{2} \Omega_{i}\left[A_{i \ell \ell}^{0 m m} U\left(U_{\ell}-m U\right)+B_{i \ell \ell}^{0 m m} V\left(V_{\ell}-m V\right)\right. \\
& \left.+C_{i \ell, \ell-1}^{0 m m *} V W_{\ell-1}+C_{i \ell, \ell+1}^{0 m m *} V W_{\ell+1}\right] d r / \int_{0}^{R} \rho_{0} r^{2}\left[U^{2}+\ell(\ell+1) V^{2}\right] d r .
\end{aligned}
$$

The functions $U_{\ell}(r), V_{\ell}(r), W_{\ell-1}(r)$ and $W_{\ell+1}(r)$ represent the simple decomposition of the vector field $i \hat{z} \times \mathbf{u}$ in terms of spheroidal and toroidal vector spherical harmonics (Vorontsov and Zharkov 1981), which contains one spheroidal field of the same degree $\ell$, and two toroidal fields of degree $\ell-1$ and $\ell+1$ :

$$
\begin{gathered}
i \hat{z} \times \mathbf{u}=U_{\ell}(r) \hat{r} Y_{\ell m}(\theta, \phi)+V_{\ell}(r) \nabla_{1} Y_{\ell m}(\theta, \phi) \\
-W_{\ell-1}(r) \hat{r} \times \nabla_{1} Y_{\ell-1, m}(\theta, \phi)-W_{\ell+1}(r) \hat{r} \times \nabla_{1} Y_{\ell+1, m}(\theta, \phi), \\
U_{\ell}=m V, \quad V_{\ell}=\frac{m}{\ell(\ell+1)}(U+V), \\
W_{\ell-1}=-i\left[\frac{(\ell+m)(\ell-m)}{\ell^{2}(2 \ell+1)(2 \ell-1)}\right]^{1 / 2}[U+(\ell+1) V],
\end{gathered}
$$




$$
W_{\ell+1}=i\left[\frac{(\ell+m+1)(\ell-m+1)}{(\ell+1)^{2}(2 \ell+1)(2 \ell+3)}\right]^{1 / 2}[U-\ell V]
$$

$U=U_{\ell m n}(r)$ and $V=V_{\ell m n}(r)$ describe the zero-order displacement field $\mathbf{u}(\mathbf{r})$. The angular integrals

$$
\begin{aligned}
A_{k J^{\prime} J}^{q M^{\prime} M} & =\int_{4 \pi} Y_{k q} Y_{J^{\prime} M^{\prime}} Y_{J M^{\prime}}^{*} d \Omega \\
B_{k J^{\prime} J}^{q M^{\prime} M} & =\int_{4 \pi} Y_{k q}\left(\nabla_{1} Y_{J^{\prime} M^{\prime}}\right) \cdot\left(\nabla_{1} Y_{J M}\right)^{*} d \Omega \\
& =\int_{4 \pi} Y_{k q}\left(-\hat{r} \times \nabla_{1} Y_{J^{\prime} M^{\prime}}\right) \cdot\left(-\hat{r} \times \nabla_{1} Y_{J M}\right)^{*} d \Omega, \\
C_{k J^{\prime} J}^{q M^{\prime} M} & =\int_{4 \pi} Y_{k q}\left(\nabla_{1} Y_{J^{\prime} M^{\prime}}\right) \cdot\left(-\hat{r} \times \nabla_{1} Y_{J M}\right)^{*} d \Omega
\end{aligned}
$$

are determined explicitly in terms of Wigner's 3-j symbols (Edmonds 1957; Luh 1973).

When $\Omega$ is a function of $r$ alone, expression (7) for the frequency splitting reduces to (see also Gough 1981)

$$
\delta \omega=m \frac{\int_{0}^{R} \rho_{0} r^{2} \Omega(r)\left[V^{2}+2 U V-U^{2}-\ell(\ell+1) V^{2}\right] d r}{\int_{0}^{R} \rho_{0} r^{2}\left[U^{2}+\ell(\ell+1) V^{2}\right] d r} .
$$

For the simplest case of uniform rotation $\Omega=\Omega_{0}$, it reduces further to

$$
\delta \omega=m \Omega_{0} \frac{\int_{0}^{R} \rho_{0} r^{2}\left(V^{2}+2 U V\right) d r}{\int_{0}^{R} \rho_{0} r^{2}\left[U^{2}+\ell(\ell+1) V^{2}\right] d r}-m \Omega_{0},
$$

the result first obtained by Cowling and Newing (1949) and Ledoux (1951). When the oscillations of the uniformly rotating star are viewed in the corotating frame, the last term in the expression (11) disappears: this term describes the simple kinematic effect of the transformation of the coordinate system to the inertial frame. The first term on the right-hand side describes the influence of the Coriolis forces.

When $\Omega=\Omega(r), \delta \omega$ is proportional to $m$; the frequency separation within a particular multiplet is uniform. This frequency separation is different for different multiplets: different modes sample the stellar interior at different depths, and the observational frequency splittings can be used to infer $\Omega$ as a function of $r$.

The latitudinal dependence of the angulal velocity of rotation does not affect the symmetry of the multiplet splitting ( $\delta \omega$ remains an odd function of $m$ ), but distorts the equidistant separation of frequencies with respect to $m$ : modes with higher $|m|$ concentrate more closely around the equatorial plane, and are thus more sensitive to the rotation near this plane. The rotational splittings thus allow one to study the internal rotation as a function of both depth and latitude. Symmetry properties of the spherical harmonics define the selection rules for the contribution of different terms of the expansion (6) to the 
frequency splitting. The angular integrals (9) differ from zero only if $q=M^{\prime}-M$ and values of $k, J^{\prime}, J$ satisfy triangle rule. Integrals $A$ and $B$ differ from zero only if $k+J^{\prime}+J$ is even; for the integral $C$ to differ from zero, $k+J^{\prime}+J$ must be odd. Only even components in the expansion (6) thus contribute to the frequency splitting: asymmetry of the stellar rotation with respect to the equatorial plane has no influence on the oscillation frequencies. Only the components with $i \leq 2 \ell$ contribute to the splitting: higher-order harmonics in the latitude dependence of the rotational velocity can not be detected with low-degree modes.

In helioseismology, reliable measurements of rotational splitting are now available for $\mathrm{p}$ modes in a wide range of degree $\ell$. For the interpretation of the odd component of the multiplet splitting, the leading-order perturbation analysis is adequate, at least within the current accuracy of the observational data. The reconstruction of the internal differential rotation from the frequency splittings thus represents a linear inverse problem, which is studied successfully with different linear inversion techniques. For the extensive literature in this area, the reader is referred to the current reviews on helioseismology (Gough and Toomre 1991; Libbrecht and Woodard 1991; Dziembowski and Goode 1991a; Gough and Thompson 1991; Vorontsov 1992).

\section{Second-order effects of rotation}

The analysis of the effects of order $\Omega^{2}$ on the oscillation frequencies is more complicated, and for realistic stellar models requires some additional simplifying assumptions. The difficulty comes from the fact that the rotational distortion of the equilibrium stellar configuration is also of order $\Omega^{2}$, and contributes to the same order to the oscillation frequencies. The rotational distortion of the star thus must be specified; this problem is very difficult in general, if the self-consistent stellar model is required to be not only in dynamical, but also in the thermal equilibrium, and the stellar model itself is an evolutionary model.

After the rotational distortion of the equilibrium configuration is specified somehow, the extension of the perturbation analysis to second order is a straightforward procedure (see e.g. Saio 1981; Vorontsov and Zharkov 1981 for uniform rotation, and Vorontsov 1981; Gough and Thompson 1990; Dziembowski and Goode 1992 for arbitrary axisymmetric differential rotation). The standard stationary perturbation technique for Hermitian operators 
in the frequency splittings is clearly seen for the high-degree $p$ and $f$ modes trapped near the stellar surface. Modes with $m=0$ can be considered as a superposition of waves travelling around the star along meridians, and modes with $m= \pm \ell$ are formed by waves travelling along the equator. Due to the asphericity induced by the rotation, the wave travel path along the equator is larger than along the meridian, and frequencies of the tesseral modes with $m= \pm \ell$ becomes larger than those of zonal modes $(m=0)$. Together with second-order effects of the Coriolis forces, the distortion of the equilibrium configuration contributes to the multiplet splitting with an asymmetric component (even function of $m$ ), because the effect is the same for waves travelling in opposite directions.

In the context of stellar oscillations, the distortion of the equilibrium configuration by the differential rotation was considered in most detail by Dziembowski and Goode (1992). The spherically-symmetric part of the distortion was ignored, because it does not contribute to the multiplet splitting, producing only an overall shift of the multiplets. For solar p modes, it was found that the distortion dominates in the second-order rotational effects (see also Gough and Thompson 1990), and the effect itself is large enough to be taken into account in the interpretation of the even component in the frequency splittings (the even component can be produced also by other aspherical effects, including magnetic field).

The applicability of the standard perturbation technique assumes the linear interaction between different modes to be small. This condition can be violated, if the unperturbed frequencies of the modes which can interact are close enough, with the frequency difference of the order of the rotational splitting. This situation is familiar in terrestrial seismology, and can be treated by an appropriate modification of the perturbational technique for the case of quasi-degeneracy (e.g. Messiah 1961). Three-mode coupling induced by uniform rotation was considered by Vorontsov and Zharkov (1981) with an application to the computation of Jovian oscillations. Dziembowski and Goode (1992) considered accidental degeneracies in the solar p-mode spectrum; the effects of mode coupling on the rotational splitting were found to be insignificant.

\section{WEAK MAGNETIC FIELD}

A large-scale magnetic field in the star can be considered weak and its effect on the stellar oscillations can be treated within a perturbation theory, if both the dynamical effects of the magnetic field on the oscillations and the magnetic distortion of the equilibrium configuration are small. For reasonable magnetic fields, these conditions are always satisfied in stellar interiors, but can be violated near the surface of the magnetic star: we shall discuss this situation shortly in the last section of the review.

The perturbation analysis can be based directly on the oscillation equations, which include the effects of magnetic field in MHD approximation (see Gough and Taylor 1984; Dziembowski and Goode 1984; Unno et al. 1989; Gough and Thompson 1990; and references therein to early work). An alternative approach is the application of the variational principle (e.g. Kovetz 1966; Lynden-Bell and Ostriker 1967). When the variational principle is used, 
the convenient symmetric form of the Lagrangian can be written as (Vorontsov 1986)

$$
\begin{aligned}
L & =\Gamma_{1} p_{0}\left(\nabla \cdot \mathbf{u}^{*}\right)(\nabla \cdot \mathbf{u})+\frac{1}{2}\left[\rho_{0}(\mathbf{u} \cdot \nabla)\left(\mathbf{u}^{*} \cdot \nabla \psi_{0}\right)+\rho_{0}\left(\mathbf{u}^{*} \cdot \nabla\right)\left(\mathbf{u} \cdot \nabla \psi_{0}\right)\right. \\
& \left.+\left(\nabla \cdot \mathbf{u}^{*}\right)\left(\mathbf{u} \cdot \nabla p_{0}\right)+(\nabla \cdot \mathbf{u})\left(\mathbf{u}^{*} \cdot \nabla p_{0}\right)\right]+\rho_{0}\left(\mathbf{u} \cdot \nabla \psi_{1}^{*}+\mathbf{u}^{*} \cdot \nabla \psi_{1}\right) \\
& +\frac{1}{4 \pi G} \nabla \psi_{1}^{*} \cdot \nabla \psi_{1}-\rho_{0} \omega^{2} \mathbf{u}^{*} \cdot \mathbf{u} \\
& +\frac{1}{4 \pi} \mathbf{H}_{1}^{*} \cdot \mathbf{H}_{1}-\frac{1}{8 \pi}\left[\left(\nabla \times \mathbf{H}_{0}\right) \times \mathbf{H}_{1} \cdot \mathbf{u}^{*}+\left(\nabla \times \mathbf{H}_{0}\right) \times \mathbf{H}_{1}^{*} \cdot \mathbf{u}\right]
\end{aligned}
$$

where $\mathbf{H}_{0}$ denotes the equilibrium magnetic field, $\mathbf{H}_{1}$ is its Eulerian perturbation

$$
\mathbf{H}_{1}=\nabla \times\left(\mathbf{u} \times \mathbf{H}_{0}\right),
$$

$\psi_{1}$ is the Eulerian perturbation of the gravitational potential. The first three lines in the Lagrangian correspond to the problem of adiabatic oscillations of a non-magnetic star; the last line describes the work done against the Lorentz forces. The application of the Rayleigh's principle gives the equation for the perturbations of the oscillation frequencies due to the influence of the magnetic field:

$$
\delta\left(\omega^{2}\right) \int \frac{\partial L}{\partial\left(\omega^{2}\right)} d v=-\int \frac{\partial L}{\partial p_{k}} \delta p_{k} d v
$$

where integrals are taken over the unperturbed spherical volume occupied by the star, "parameters" $p_{k}$ denote the equilibrium values of density $\rho_{0}$, pressure $p_{0}$, gravitational potential $\psi_{0}$, adiabatic exponent $\Gamma_{1}$, components of $\mathbf{H}_{0}$ and their spatial derivatives. If there is no axial symmetry of the magnetic field, or if the axis of symmetry differs from the axis of the spherical coordinate system which was chosen, zero-order eigenfunctions $\mathbf{u}$ are linear combinations of spheroidal components with different values of $m$; the coefficients are determined from the requirement that $\delta\left(\omega^{2}\right)$ is to be stationary under their variations.

Both the frequency perturbations and the magnetic distortion of the equilibrium configuration (with the only exception of force-free field, when there is no distortion) are proportional to $H^{2}$ in the leading order, and some simplifying assumptions about the equilibrium configuration must enavitably be introduced (see Gough and Thompson 1990 for a discussion). The magnetic splitting of frequency multiplets is described by an even function of $m$, because the influence of the magnetic field is the same for modes travelling in opposite directions around the star.

In helioseismology, the possible effects of a large-scale magnetic field concentrated in the solar interior were studied by Dziembowski and Goode (1984), Gough and Taylor (1984), Roberts and Campbell (1986), and in more detail by Gough and Thompson (1990). The effects of a toroidal field concentrated in a thin layer near the base of the convection zone were analized by Gough and Thompson (1988a, 1990) and Vorontsov (1988).

In the frequency splitting of solar $p$ modes, the even component is really found to be significant (Jefferies et al. 1988; Libbrecht 1989; Libbrecht and Woodard 1990). Gough and Thompson (1988b) found evidence that the original even coefficients measured by Duvall et al. (1986) were due to 
a perturbation near the surface and attributed it to a fibril magnetic field. The analysis of this even component in recent observations indicates strongly that the dominant contribution comes from a perturbation localized near the solar surface, and this perturbation varies with the solar activity cycle (Libbrecht and Woodard 1990). By the inversion of the even component, Dziembowski and Goode $(1989,1991 \mathrm{~b})$ found evidence for a small additional source of perturbation, which they attribute to a steady megagauss toroidal magnetic field localized near the base of the convection zone. The dominant near-surface perturbation was attributed by Dziembowski and Goode (1991b) to the effects of a fibril magnetic field, discussed by Zweibel and Däppen (1989) (for the discussion of the effects of fibril field, see also Bogdan 1989; Bogdan and Cattaneo 1989; and references therein).

The prominent variations of the average frequencies of solar $p$-modes during the solar cycle have been also detected (Elsworth et al. 1990; Libbrecht and Woodard 1990; Woodard and Libbrecht 1991), which also indicate a perturbation localized near the surface (Libbrecht and Woodard 1990). The effects of the chromospheric magnetic field (Campbell and Roberts 1989; Evans and Roberts 1990, 1991, 1992; Jain and Roberts 1992; Wright and Thompson 1992) and of the photospheric flux tubes (Goldreich et al. 1991) were analyzed to explain these frequency variations.

The possible effects of a large-scale magnetic field in the oscillations of variable white dwarfs were discussed by Jones et al. (1989).

\section{JOINT EFFECTS OF THE ROTATION AND MAGNETISM}

The discovery of rapid oscillations of the rotating magnetic Ap stars (see Kurtz 1990 for a review) stimulated an extensive study of the joint effects of both the rotation and magnetism, when the magnetic axis is inclined to the axis of rotation (Gough and Taylor 1984; Dziembowski and Goode 1984, 1985, 1986; Kurtz and Shibahashi 1986; Gough and Thompson 1990; for a review of theoretical work, see Shibahashi 1991). The intriguing property of the oscillations of Ap stars is that the pulsation amplitudes correlate with the phase of the magnetic field in such a way that the oscillations might be interpreted as axially symmetric $(m=0)$ dipole $(\ell=1)$ p modes with symmetry axis coincident with the magnetic axis, which is oblique to the axis of stellar rotation.

In the theoretical interpretation which is now widely accepted, the uniform rotation and global dipole magnetic field are assumed; the distortion of the equilibrium spherical configuration is neglected. To study the oscillations, it is convenient to use the corotating frame, in which the magnetic field is stationary. If the variational principle is applied, the modification of the Lagrangian (12) by the effects of rotation reduces to the appearance of the simple additional term $2 \rho_{0} \omega \Omega_{0} \mathbf{u}^{*} \cdot(i \hat{z} \times \mathbf{u})$, which describes the influence of the Coriolis forces; the angular velocity of rotation should be considered as one of the parameters $p_{k}$, with variation from 0 to $\Omega_{0}$. Joint effects of the Coriolis forces and Lorentz forces lead to the splitting of a degenerate multiplet into $2 \ell+1$ frequency components, with zero-order eigenfunctions having a mixed character (linear combinations of spheroidal vector fields with different values of $m$ ). When observed from the inertial frame, each of these modes produces 
$2 \ell+1$ frequency components, separated by the angular velocity of rotation. The frequencies of different modes can be different in the corotating frame, and the degenerate multiplet can thus be split, in general, into $(2 \ell+1)^{2}$ components. Although there is only $(2 \ell+1)$-fold degeneracy in the non-rotating non-magnetic star, such a multiple splitting arises because the perturbation is not stationary in the inertial frame.

But only the equidistant triplet structures are usually observed in the power spectra of rapidly oscillating Ap stars. To resolve this problem, Dziembowski and Goode $(1985,1986)$ argue the possibility of the selective exitation in the presence of the relatively strong magnetic field, so that only one mode is actually excited, which appears with $2 \ell+1$ frequency components in the observations from the inertial frame. The relative amplidudes of the different components depend on the relative contribution of the effects of magnetic field and rotation, and thus the mean strength of the magnetic field in the stellar interior can be measured.

Quite recently, new observations of a rapidly oscillating Ap star HR 3831 revealed that the apparent triplet structure of its oscillation frequencies is really a part of a septuplet (Kurtz et al. 1992). The interpretation of this complicated structure as a contribution of the lower-amplitude signal from the first-order correction to the velocity field, which contains a spheroidal vector harmonic of degree $\ell=3$, is discussed in these proceedings by Shibahashi and Takata (1992).

\section{"RAPID" ROTATION}

When the angular velocity of stellar rotation becomes relatively high, of the order of the oscillation frequency, Coriolis forces become of the same order as inertial forces, and can no longer be considered as a small perturbation. We meet this situation when studying the low-frequency modes; the rotation itself is usually considered to be slow enough for the centrifugal distortion of the equilibrium stellar configuration to remain relatively small.

By analogy with pressure perturbations, which provide the restoring forces for acoustic waves and global p modes, and with buoyancy forces, which provide the restoring forces for gravity waves and global $g$ and $f$ modes, the Coriolis forces represent a new type of restoring force for waves of quite different physical nature, which can also be trapped in the star and form new global modes.

In contrast with pressure perturbations, which are usually considered to be isotropic, and with buoyancy forces, which are always vertical, the geometry of Coriolis forces makes the classification of new waves somewhat more complicated.

The first class of possible motions is represented by Rossby waves, familiar in geophysics (e.g., Pedlosky 1979). These waves have predominantly horizontal displacements, and their origin is due to the latitudinal dependence of the distribution of the vertical component of the specific angular momentum in the rotating star. The curvature of the level surfaces is thus crucial for the existence of Rossby waves; corresponding pressure and density perturbations are relatively small. When viewed in the corotating frame for a star with 
uniform rotation, Rossby waves propagate in the direction opposite to the rotation (retrograde waves).

Waves of another type can appear, with significant vertical displacements, which can thus be significantly affected by buoyancy. If the stratification is convectively neutral (no buoyancy forces), these modes have a mixed character: they are a combination of pure inertial waves, which arise if the Boussinesq approximation is adequate, and prograde waves (generalized Rossby waves) with restoring forces produced by additional vorticity perturbations, which are due to Lagrangian density perturbations in the rotating star (an excellent local analysis of waves in rotating stars can be found in monograph of Unno et al. 1989; see also Ando 1989). When the stratification is convectively stable, the inertial waves are modified by buoyancy, but their frequencies remain real in the adiabatic approximation (purely oscillatory motions). In convectively unstable layers, however, the situation is much more interesting. In the linear adiabatic approximation, the wave amplitude can grow exponentially with time. The physical meaning of this phenomenon is related with the stabilizing effect of the rotation on the convection. The interaction of the inertial waves with unstable convective modes ( $\mathrm{g}^{-}$modes) can produce overstable, or even oscillatory convective motions.

In the global analysis of the long-period oscillations of rotating stars, the main difficulty is due to the fact that a particular mode can no longer be described by a single spherical harmonic $Y_{\ell m}(\theta, \phi)$. When the effects of rotation are small, the simple perturbational analysis of $p, f$ and $g$ modes shows that even if we neglect the centrifugal distortion and consider only the uniform rotation, the first-order correction to the eigenfunction of a normal mode described by a spherical harmonic $Y_{\ell m}(\theta, \phi)$ contains toroidal vector fields (equation (2)) of degree $\ell-1$ and $\ell+1$, the second-order correction includes spheroidal fields of degree $\ell-2$ and $\ell+2$, and so on. When the effects of rotation are small, this linear interaction between modes of different degree, induced by the rotation, is usually small (if there are no accidental resonances between interacting modes), and the perturbational expansion converges rapidly. But that is not necessary the case for "rapid" rotation (low-frequency modes).

The breaking of the spherical symmetry thus prevents the possibility of simple variable separations (except in the case of a uniform incompressible fluid, see Bryan 1889); only the axial symmetry remains (which is always considered to be the case), so that modes can still be identified with unique values of azimuthal order $m$. Separation of spatial variables by using the vector spherical harmonics (equations $(1,2)$ ) results in two infinite chains of coupled differential equations, with spheroidal displacements described by $\ell=|m|,|m|+2, \ldots$ ("even" modes) and $\ell=|m|+1,|m|+3, \ldots$ ("odd" modes). Such a decoupling into two series is due to the symmetry of the equilibrium configuration that remains with respect to the equatorial plane; "even" modes are symmetric, and "odd" modes are antisymmetric with respect to the equatorial plane, and modes from different series do not interact.

In this situation, different approximate techniques are used to study the low-frequency modes, which permits us at least to understand their qualitative behaviour. The most common techniques are (i) the truncation of the infinite chains of differential equations, and (ii) the application of asymptotic analysis (in sense of considering either the slow rotation or modes with high radial order). The "traditional approximation" (which neglects 
the horizontal component of the angular velocity of rotation) simplifies the problem significantly (e.g. Unno et al. 1989). The variational principle can also be generalized to study the oscillations of rotating stars (Lynden-Bell and Ostriker 1967; Ipser and Lindblom 1991).

The first prominent effect of Coriolis forces is that the degeneracy of the oscillation spectrum of a non-rotating star with respect to toroidal displacement fields (defined by equation (2): "trivial" modes with zero frequencies) is lifted. For a star with uniform rotation, these fields represent the leading term of the eigenfunctions of new modes, which are global Rossby waves with frequencies

$$
\omega=\frac{2 m \Omega}{\ell(\ell+1)}-m \Omega+O\left(\Omega^{3}\right)
$$

in the inertial frame. In the theory of stellar oscillations, these modes were first studied by Papaloizou and Pringle (1978) and were called " $r$ modes", and by Provost, Berthomieu and Rocca (1981) ("quasi-toroidal modes"). Papaloizou and Pringle (1978) used a technique of consequtive approximations, by putting the vertical displacements and Eulerian pressure perturbations to be zero in the leading approximation; Provost, Berthomieu and Rocca (1981) used an asymptotic technique with the rotational frequency as a small parameter. The asymptotic analysis was extended by Smeyers, Craeynest and Martens (1981) to account for gravity perturbations. All the studies are largely limited by the uniform rotation; differential rotation can lead to the Kelvin-Helmholtz instability (shear instability), and makes the problem much less tractable (Papaloizou and Pringle 1978; Dziembowski and Kosovichev 1987a,b).

An interesting property of $\mathrm{r}$ modes can be seen directly from expression (15): their frequencies are degenerate, at least in the leading order, with respect to the radial distribution of the eigenfunctions, i.e. with respect to the radial order $n$. With the only exeption of convectively neutral stratification, this degeneracy is lifted when higher-order terms of the asymptotic expansions are taken into account. It was demonstrated by Provost, Berthomieu and Rocca (1981), by using polytropic models, that the eigenfrequencies decrease with radial order when the stratification is convectively stable (polytrope of index 3 ), and increase with radial order when the stratification is convectively unstable (polytrope of index 1 ). If the absolute value of the buoyancy frequency is of the order of $\Omega$ or smaller, the asymptotic analysis becomes invalid. Even for a slowly rotating star, this situation can occure in the region with effective convection, and was analized in detail by Dziembowski and Kosovichev (1987a): a technique was developed which uses matching of the asymptotic solutions with the direct numerical solutions of the (truncated) oscillation equations in this region. For differential rotation with $\Omega=\Omega(r)$, Dziembowski and Kosovichev (1987a) argue the possibility of r-mode trapping in the vicinity of the local minimum of $\Omega(r)$.

Although the velocity field of $r$ modes is horizontal and divergence-free in the leading order, higher-order terms in the eigenfunctions produce some pressure perturbations, and modes can be excited by the destabilizing effect of $\kappa$ mechanism. Non-adiabatic analyses of Saio (1982) and Berthomieu and Provost (1983) indicate that $\mathrm{r}$ modes can become overstable in ZZ Ceti stars. 
The asymptotic properties of high-order gravity modes ( $\mathrm{g}^{+}$modes) in the presence of rotation were studied by Berthomieu et al. (1978). It was shown that the "traditional approximation" is applicable for these low-frequency modes with predominantly horizontal displacements. Results of the trancated analysis of the high-order $g$ modes (Lee and Saio 1987a) were found to be in qualitative agreement with the asymptotic approach. Using the asymptotic analysis in "traditional approximation", Lee and Saio (1987a) demonstrated the appearance of the low-frequency cut-off of the high-order gravity modes, which is due to the stabilizing effect of the Coriolis forces, acting as additional restoring forces.

But much more interesting is the influence of rotation on the unstable gravity modes ( $\mathrm{g}^{-}$modes, or convective modes), which can be considered as an interaction between convective modes and inertial waves governed by the rotation, and describes (in general) the interaction between convection, rotation and pulsation. This problem has been an object of intensive study in recent years.

The stabilizing effect of Coriolis forces on convection makes the unstable convective modes become overstable, or even purely oscillatory. The concept of "negative-energy" modes becomes very productive in the physical understanding of the related phenomena (Lee and Saio 1989, 1990c).

If an overstable convective mode is completely stabilized by the rotation, two purely oscillatory modes appear, one with positive and another with negative energy. The kinetic energies of both modes are surely positive in the corotating frame (the studies are largely limited to uniform rotation), but one of these modes has negative kinetic energy when considered in the inertial frame: the kinetic energy of the equilibrium rotating flow is larger than that of the rotating and oscillating flow. Equipartition of kinetic and potential energies persists in the inertial frame (Lee and Saio 1990c), and the total energy of the mode is thus negative.

If energy is extracted from the negative-energy mode, its amplitude grows; the energy source is provided by the star. The radiative dissipation destabilizes the negative-energy mode, making the purely oscillatory motion overstable (Lee and Saio 1987b). Due to the resonance coupling of the convective modes in the stellar core with the stable envelope $g$ modes, the overstable low-frequency modes can appear in rotating massive main-sequence stars (Osaki 1974; Lee and Saio 1986, 1987b, 1989, 1990c; Lee 1988).

The line-profile variations produced by low-frequency $g$ modes excited by the oscillatory convective modes in a rapidly rotating massive main-sequence star were studied by Lee and Saio (1990a) and Lee, Jeffery and Saio (1992).

Inertial oscillations of Jupiter were studied by Lee and Saio (1990b) and Lee, Strohmayer and Van Horn (1992) as an explanation of the slowly oscillating thermal features discovered in the Jovian atmosphere by Magalhães et al. (1989) and Deming et al. (1989).

\section{"STRONG" MAGNETIC FIELD}

Even when the overall magnetic field is relatively weak, the analysis of magnetic effects in stellar oscillations by the standard perturbation technique can become invalid near the surface, if the magnetic field does not vanish 
there. Because of the low density, the magnetic pressure in the surface layers can become of the same order as gas pressure, and the Lorentz forces can become of the same order as inertial forces. The perturbation analysis then becomes invalid locally; this situation is exactly what we expect for the rapidly oscillating magnetic Ap stars.

The first steps in the improvement of the theoretical description were taken by Biront et al. (1982) using a singular perturbation technique (method of matched asymptotic expansions, which originates from boundary layer theory). The analysis of Biront et al. (1982) was limited to the study of the dynamical distortion of the velocity field in the outer stellar layers. This analysis was developed further by Roberts and Soward (1983) and Campbell and Papaloizou (1986), who included the energy dissipation through Alfvénictype waves which are generated by the pulsations.

Despite of these efforts, the theoretical understanding of the rapid oscillations in Ap stars is far from being complete; there is no convincing description of the selective mode excitation, although it has been quite evident for the last ten years that the magnetic field plays a crucial role in these oscillations. Much work is needed to understand the excellent observational data which are already available, and which will undoubtedly be refined by forthcoming observations.

Author thanks Douglas Gough for carefully reading the manuscript to improve English, and for comments.

\section{REFERENCES}

Ando, H. 1989, Publ. Astron. Soc. Japan, 41, 289.

Berthomieu, G., and Provost, J. 1983, Astr. Ap., $122,199$.

Berthomieu, G., Gonczi, G., Graff, Ph., Provost, J., and Rocca, A. 1978, Astr. Ap., 70, 597.

Biront, D., Goossens, M., Cousens, A., and Mestel, L. 1982, M.N.R.A.S., 201, 619.

Bogdan, T. J. 1989, Ap. J., 345, 1042.

Bogdan, T. J., and Cattaneo, F. 1989, Ap. J., $342,545$.

Bryan, G. H. 1889, Philos. Trans. R. Soc. London, A 180, 187.

Campbell, C. G., and Papaloizou, J. C. B. 1986, M.N.R.A.S., 220, 577.

Campbell, W. R., and Roberts, B. 1989, Ap. J., 338, 538.

Cowling, T. G., and Newing, R. A. 1949, Ap. J., 109, 149.

Cox, J. P. 1980, Theory of Stellar Pulsation (Princeton: Princeton University Press).

Cuypers, J. 1980, Astr. Ap., 88, 207.

Deming, D., Mumma, M. J., Espenak, F., Jennings, D. E., Kostiuk, T., Wiedemann, G., Loewenstein, R., and Piscitelli, J. 1989, Ap. J., 343, 456.

Durney, B. R., Hill, F., and Goode, P. R. 1988, Ap. J., 326, 486.

Duvall, T. L., Jr., Harvey, J. W., and Pomerantz, M. A. 1986, Nature, 321, 500.

Dziembowski, W. A., and Goode, P. R. 1984, Mem. Soc. Astron. Ital., 55, 185.

Dziembowski, W. A., and Goode, P. R. 1985, Ap. J. (Letters), 296, L27. 
Dziembowski, W. A., and Goode, P. R. 1986, in: Seismology of the Sun and the Distant Stars, ed. D. O. Gough (Dordrecht: Reidel), p.441.

Dziembowski, W. A., and Goode, P. R. 1989, Ap. J., 347, 540.

Dziembowski, W. A., and Goode, P. R. 1991a, in: Solar Interior and Atmosphere, eds A. N. Cox, W. C. Livingston and M. S. Matthews (Tucson: University of Arizona Press), p.501.

Dziembowski, W. A., and Goode, P. R. 1991b, Ap. J., 376, 782.

Dziembowski, W. A., and Goode, P. R. 1992, Ap. J., in press.

Dziembowski, W., and Kosovichev, A. 1987a, Acta Astronomica, 37, 313.

Dziembowski, W., and Kosovichev, A. 1987b, Acta Astronomica, 37, 341.

Edmonds, A. R. 1957, Angular Momentum in Quantum Mechanics (Princeton: Princeton University press).

Elsworth, Y., Howe, R., Isaak, G. R., McLeod, C. P., and New, R. 1990, Nature, 345, 322.

Evans, D. J., and Roberts, B. 1990, Ap. J., 356, 704.

Evans, D. J., and Roberts, B. 1991, Ap. J., 371, 387.

Evans, D. J., and Roberts, B. 1992, Nature, 355, 230.

Goldreich, P., Murray, N., Willette, G., and Kumar, P. 1991, Ap. J., 370, 752.

Gough, D. O. 1981, M.N.R.A.S., 196, 731.

Gough, D. O., and Kosovichev, A. G. 1992, these proceedings.

Gough, D. O., and Taylor, P. P. 1984, Mem. Soc. Astron. Ital., 55, 215.

Gough, D. O., and Thompson, M. J. 1988a, in: Advances in Helio- and Asteroseismology, eds J. Christensen-Dalsgaard and S. Frandsen (Dordrecht: Reidel), p.155.

Gough, D. O., and Thompson, M. J. 1988b, in: Advances in Helio- and Asteroseismology, eds J. Christensen-Dalsgaard and S. Frandsen (Dordrecht: Reidel), p.175.

Gough, D. O., and Thompson, M. J. 1990, M.N.R.A.S., 242, 25.

Gough, D. O., and Thompson, M. J. 1991, in: Solar Interior and Atmosphere, eds A. N. Cox, W. C. Livingston and M. S. Matthews (Tucson: University of Arizona Press), p.519.

Gough, D., and Toomre, J. 1991, Ann. Rev. Astron. Astrophys., 29, 627.

Hansen, C. J., Cox, J. P., and Van Horn, H. M. 1977, Ap. J., 217, 151.

Ipser, J. R., and Lindblom, L. 1991, Ap. J., 378, 285.

Jain, R., and Roberts, B. 1992, Ap. J., submitted.

Jefferies, S. M., Pomerantz, M. A., Duvall, T. L., Jr., Harvey, J. W., and Jaksha, D. B. 1988, in: Seismology of the Sun and the Sun-Like Stars, ed. E. J. Rolfe (ESA SP-286; Noordwijk: ESA), p.279.

Jones, P. W., Pesnell, W. D., Hansen, C. J., and Kawaler, S. D. 1989, Ap. J., 336, 403.

Kovetz, A. 1966, Ap. J., 146, 462.

Kurtz, D. W. 1990, Ann. Rev. Astron. Astrophys., 28, 607.

Kurtz, D. W., Kanaan, A., Martinez, P., and Tripe, P. 1992, M.N.R.A.S., 255, 289.

Kurtz, D. W., and Shibahashi, H. 1986, M.N.R.A.S., 223, 557.

Ledoux, P. 1951, Ap. J., 114, 373.

Lee, U. 1988, M.N.R.A.S., $232,711$.

Lee, U., and Saio, H. 1986, M.N.R.A.S., 221, 365.

Lee, U., and Saio, H. 1987a, M.N.R.A.S., 224, 513.

Lee, U., and Saio, H. 1987b, M.N.R.A.S., $225,643$. 
Lee, U., and Saio, H. 1989, M.N.R.A.S., 237, 875.

Lee, U., and Saio, H. 1990a, Ap. J., 349, 570.

Lee, U., and Saio, H. 1990b, Ap. J. (Letters), 359, L29.

Lee, U., and Saio, H. 1990c, Ap. J., 360, 590.

Lee, U., Jeffery, C. S., and Saio, H. 1992, M.N.R.A.S., 254, 185.

Lee, U., Strohmayer, T. E., and Van Horn, H. M. 1992, preprint.

Libbrecht, K. G. 1989, Ap. J., 336, 1092.

Libbrecht, K. G., and Woodard, M. F. 1990, Nature, 345, 779.

Libbrecht, K. G., and Woodard, M. F. 1991, Science, 253, 152.

Luh, P. C. 1973, Geophys. J., 32, 187.

Lynden-Bell, D., and Ostriker, J. P. 1967, M.N.R.A.S., 136, 293.

Magalhães, J. A., Weir, A. L., Conrath, B. J., Gierasch, P. J., and Leroy, S. S. 1989, Nature, 337, 444.

Messiah, A. 1961, Quantum Mechanics (Amsterdam: North-Holland Publ.).

McDermott, P. N., Van Horn, H. M., and Hansen, C. J. 1988, Ap. J., 325, 725.

Osaki, Y. 1974, Astr. Ap., 189, 469.

Papaloizou, J., and Pringle, J. E. 1978, M.N.R.A.S., 182, 423.

Pedlosky, J. 1979, Geophysical Fluid Dynamics (Berlin: Springer-Verlag).

Provost, J., Berthomieu, G., and Rocca, A. 1981, Astr. Ap., 94, 126.

Roberts, B., and Campbell, W. R. 1986, Nature, 323, 603.

Roberts, P. H., and Soward, A. M. 1983, M.N.R.A.S., 205, 1171.

Saio, H. 1981, Ap. J., 244, 299.

Saio, H. 1982, Ap. J., 256, 717.

Shibahashi, H. 1991, in: Challenges to Theories of the Structure of ModerateMass Stars, eds D. Gough and J. Toomre (Berlin: Springer), p.393.

Shibahashi, H., and Takata, M. 1992, these proceedings.

Smeyers, P., Craeynest, D., and Martens, L. 1981, Astrophys. Space Sci., 78, 483.

Strohmayer, T., Ogata, S., Iyetomi, H., Ichimaru, S., and Van Horn, H. M. 1991, Ap. J., 375, 679 .

Unno, W., Osaki, Y., Ando, H., Saio, H., and Shibahashi, H. 1989, Nonradial Oscillations of Stars (2nd ed.; Tokyo: University of Tokyo Press).

Vorontsov, S. V. 1981, Astron. Zh., 58, 1275.

Vorontsov, S. V. 1986, in: Upper Main Sequence Stars with Anomalous Abundances, eds C. R. Cowley etal. (Dordrecht: Reidel), p.37.

Vorontsov, S. V. 1988, in: Advances in Helio- and Asteroseismology, eds J. Christensen-Dalsgaard and S. Frandsen (Dordrecht: Reidel), p.151.

Vorontsov, S. V. 1992, Astron. Zh., in press.

Vorontsov, S. V., and Zharkov, V. N. 1981, Astron. Zh., 58, 1101.

Woodard, M. F., and Libbrecht, K. G. 1991, Ap. J. (Letters), 374, L61.

Wright, A. N., and Thompson, M. J. 1992, Astr. Ap., in press.

Zweibel, E. G., and Däppen, W. 1989, Ap. J., 343, 994. 